\title{
Two-wave photon Doppler velocimetry measurements in direct impact Hopkinson pressure bar experiments
}

\author{
Lewis J. Lea a and Andrew P. Jardine \\ SMF Fracture and Shock Physics Group, Cavendish Laboratory, JJ Thomson Avenue, Cambridge CB3 0HE, UK
}

\begin{abstract}
Direct impact Hopkinson pressure bar systems offer many potential advantages over split Hopkinson pressure bars, including access to higher strain rates, higher strains for equivalent striker velocity and system length, lower dispersion and faster achievement of force equilibrium. Currently advantages are gained at a significant cost: the fact that input bar data is unavailable removes all information about the striker impacted specimen face, preventing the determination of force equilibrium, and requiring approximations to be made on the sample deformation history. Recently photon Doppler velocimetry methods have been developed, which can replace strain gauges on Hopkinson bars. In this paper we discuss an experimental method and complementary data analysis for using Doppler velocimetry to measure surface velocities of the striker and output bars in a direct impact bar experiment, allowing similar data to be recorded as in a split bar system, with the same level of convenience. We discuss extracting velocity and force measurements, and improving the accuracy and convenience of Doppler velocimetry on Hopkinson bars. Results obtained using the technique are compared to equivalent split bar tests, showing improved stress measurements for the lowest and highest strains.
\end{abstract}

\section{Introduction}

Two general types of Hopkinson Bar systems exist for compression experiments: Split Hopkinson Pressure Bars (SHPBs, also known as Kolsky Bars [1]) and Direct Impact systems (DIHBs) [2]. In a typical SHPB test, a sample is placed between an input and output bar as depicted in Fig. 1 (a). The input bar, B, is struck by a striker bar, A, passing a pressure wave through the input bar, into the sample and out through the output bar, C. A fourth bar, D, traps the momentum transferred by the striker, protecting the experiment. The local strain at some point on the input and output bars is typically measured using strain gauges. The bars remain elastic during the experiment, allowing the force and velocity histories of the end faces of the bars, and therefore the sample, to be found from contact measurements at a distance.

In direct impact bars, the input bar is removed and the sample is struck directly by the striker bar as shown in Fig. 1 (b). Striking the sample directly has many benefits over using an input bar. Higher strains be achieved with an equivalent system as the striker bar unloads over multiple passes of the wave, rather than one in an SHPB. Higher strain rates can be achieved as striking the sample directly bypasses the collision between the striker and input bar, which limits the firing velocity based on the properties of the bars,

$$
V_{\text {max }}=\frac{Y_{b a r}}{Z_{b a r}}
$$

where $Y_{b a r}$ is the yield stress of the bars and $Z_{b a r}$ is their acoustic impedance. In a DIHB experiment the striker decelerates more slowly as it continues moving with the

\footnotetext{
${ }^{a}$ Corresponding author: 11379@cam.ac.uk
}

(a)

(b)

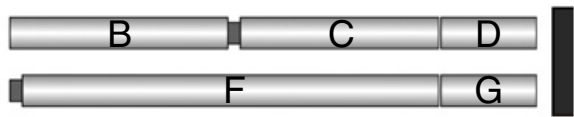

Figure 1. The co-axial bar arrangements in typical SHPB (a) and DIHB (b) systems. The experiment is initiated by the striker bars, A and E respectively, colliding with the system.

deforming sample, and does so for multiple passes of the wave, meaning the peak and average stresses experienced by the striker bar for a given velocity are lower, and higher shot velocities can be used given the same bar material.

The removal of the input bar substantially reduces the length of the system, making direct impact systems more convenient to use and the geometry also removes the Pochhammer-Chree dispersion that occurs as the pulse travels though the input bar [3]. Some specimen types may reach force equilibrium faster in a DIHB setup as removing dispersion makes the rising edge of the stress pulse steeper, equilibrium is still however dominated by the elastic properties of the specimen.

Currently, the advantages gained in a direct impact setup come at a significant cost. Removing the input bar removes all gauge information about the input face, meaning approximations have to be made about the striker face velocity and therefore strain and strain rate. Similarly the time at which sample equilibrium is reached cannot be experimentally verified. High speed photography has been used since the earliest direct impact experiments to measure the velocity of the striker bar [2] however this provides no information about the force at the striker face, has limited resolution and is time consuming. Progress has recently been made using strain gauges to instrument striker bars [4]. However these measure the particle

This is an Open Access article distributed under the terms of the Creative Commons Attribution License 4.0, which permits unrestricted use, distribution, and reproduction in any medium, provided the original work is properly cited. 


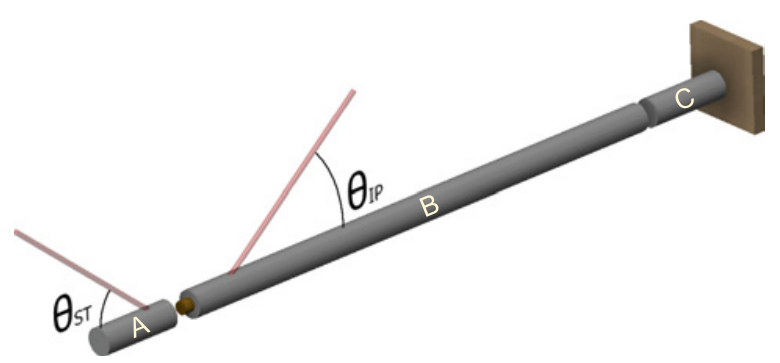

Figure 2. The incidence angles between the probing lasers and the bar axis in a DIHB system. The spot probing the striker bar, $\mathrm{A}$, is positioned based on the location of the bar at the moment of impact.

velocity in the rest frame of the striker bar, providing no direct information on the bulk velocity of the bar, and strain gauges remain susceptible to damage even in specialised barrels. Both methods make DIHB experiments much less convenient to perform, both during the experiment and in post-processing, sacrificing one of the main attractions of this technique.

Recently, Photon Doppler Velocimetry (PDV) methods have been developed by Avinadav et al. [5] which provide equivalent sets of measurements to those made by strain gauges on an SHPB. This is achieved by making a noncontact measurement of the surface velocity of the bar through reflecting a laser beam off the surface. The source and returning light are combined, creating a time varying interference pattern from which the velocity history of a bar can be deduced. These methods measure local velocity, not strain, making them well suited to obtaining data from a striker bar during a direct impact experiment where there are velocity components due to bulk motion and elastic deformation.

In this paper we will demonstrate the use of PDV to measure both the velocity and force on the striker face of a direct impact bar specimen, allowing data to be collected that is similar to that obtained using an SHPB, with similar convenience. These results are compared with traditional direct impact analysis of the same data and with SHPB tests under the same conditions. Finally we will discuss general methods for improving the convenience and accuracy of all PDV-based bar systems.

\section{Experimental techniques}

\subsection{The bar systems}

The DIHB system was used in a standard arrangement [2], depicted in Fig. 2. The bars were a Dural alloy, $25 \mathrm{~mm}$ in diameter, the output bar, B, was $1 \mathrm{~m}$ long and the striker, A, was $25 \mathrm{~cm}$ long. The sample of interest was attached to the face of the output bar using Vaseline lubricant.

The SHPB system consisted of 12.7 diameter mm bars, with $50 \mathrm{~cm}$ input and output bars, and a $25 \mathrm{~cm}$ striker bar. Vaseline was similarly used to provide an equivalent test. In both cases the bars were mounted coaxially on V-blocks and the striker was propelled using a gas gun. The striker velocity before impact was measured using a pair of light gates, the second of which was placed such that it was

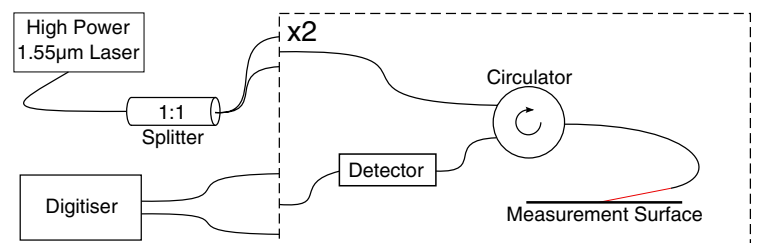

Figure 3. The photon Doppler interforometer: Light is passed by the circulator to the measurement surface, and then from the surface to $\mathrm{GHz}$ response photodiodes, which output an intensity proportional voltage. Interferometer probes were orientated as shown in Fig. 2.

partially obscured by the test sample and was and used to trigger the optical diagnostics.

\subsection{Optical velocity measurements}

A standard [6] $1.55 \mu \mathrm{m}$ optical fibre interferometer, depicted in Fig. 3, was used to probe the bar surfaces. Light reflecting off of the bar surface is classically Doppler shifted by

$$
\Delta f=\frac{2 V}{\lambda} .
$$

Unshifted light is partially reflected back at the cleaved fibre end, and added to surface reflected light collected by the same fibre. The combination creates an interference pattern with beat frequency dependant on the shift. The entire pattern is modulated by a faster component averaged out during measurement. The resultant measured intensity profile is

$$
I(t)=I_{0}+I_{1} \cos (2 \pi \Delta f t),
$$

where $I_{0}$ and $I_{1}$ are dependant on many variables including the incident intensity of the laser and the reflectivity of the surface. However they are unimportant, as all required information is encoded in the frequency of the pattern. Frequency measurement makes the technique resilient to typical noise.

Velocity histories of the faces of the striker bar and output bar in contact with the sample were deduced from surface velocity measurements made further down the bars as depicted in Fig. 2. Surface velocities were extracted from the PDV data using a standard procedure [6]. Each interference pattern, exemplified in Fig. 4 (a) was split into a series of overlapping time windows, each of which were fast fast Fourier transformed, creating a series of spectra, depicted in Fig. 4 (b), where each vertical slice is a windowed spectrum. The final velocity measurements are made by treating the spectra as probability density functions and fitting a Gaussian curve to each window's spectrum. Background spectra are removed and regions of interest are defined to reduce the effects of noise on the measurement. A strong signal near zero velocity, visible in Fig. 4 (b), caused by imperfections in the optical components is both subtracted and avoided during the selection of regions of interest. Interpolation is used to fit a curve between the mean points of the Gaussian curves fit to each window. Window lengths of $8.2 \mu$ s were used with a $7.2 \mu$ s overlap, giving a $2.1 \mu$ s step size. 
(a)

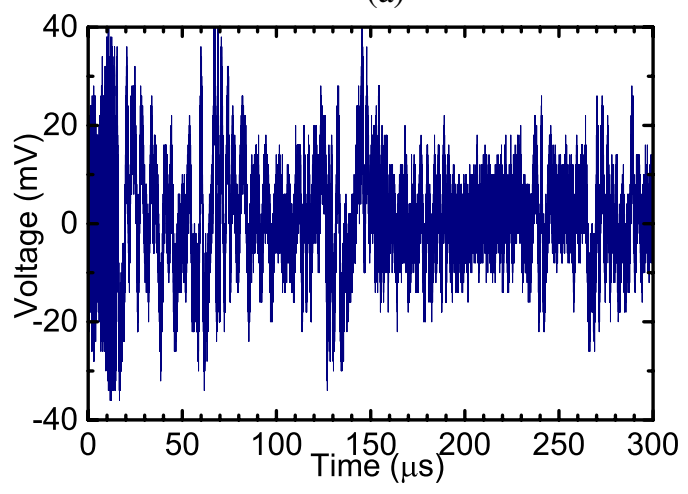

(b)

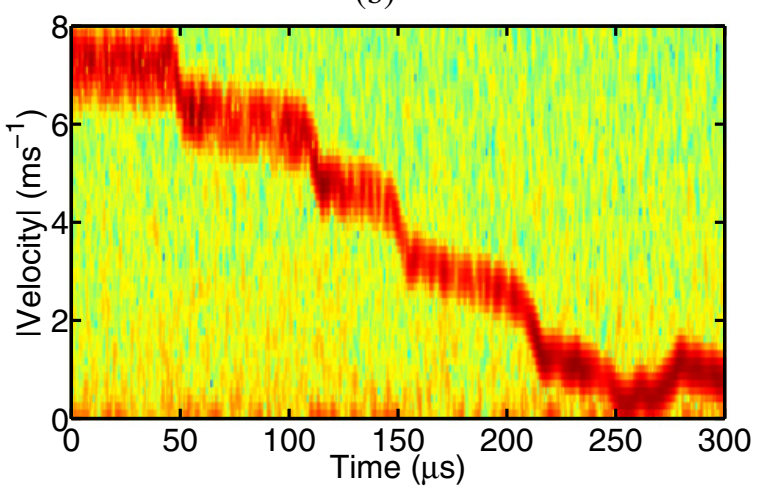

Figure 4. (a) The raw interferance pattern measured at the photodiode in the photon Doppler interferometer. (b) The probability map of the velocities obtained from Fourier transforming segments of the interferance pattern as described in the body text. The velocities depicted relate to the frequencies measured as defined by Eqs. (2) and (3).

True velocities are found by accounting for the the projection of the bar axis onto the laser axis

$$
V_{\text {true }}=\frac{V_{\text {meas }}}{\cos \theta}
$$

where $\theta$ is incidence angle of the probing laser depicted in Fig. 2. Light gate measurements were used to confirm the measurement of striker velocity immediately before impact, which is required for correct analysis as shown in Sect. 3.

\subsection{Input \& output bar measurements}

The strain gauges usually placed on the input and output bars were replaced with retro-reflective paint, which consists of a layer of small glass spheres that cause incident laser light to reflect back on itself. Retro reflection allowed the fibres to be placed at glancing angles (typically $10^{\circ}$ ) with reference to the bar surface without the severe loss of light that normally occurs in diffuse reflection. Glancing angle measurements lead to a reduction in errors caused by the precision of the laser incidence angle and bending velocities during the experiment.

Bending errors are quantified by pointing fibres at opposite sides of the same point on the bar, allowing the measurement projections to be separated into axial and radial (bending) velocities [7]. Bending modes cause a velocity towards one probe, and way from the other, resulting in the measured velocities

$$
V_{1}=V_{\text {axial }} \cos \theta+V_{\text {bend }} \sin \theta,
$$

and

$$
V_{2}=V_{\text {axial }} \cos \theta-V_{\text {bend }} \sin \theta,
$$

allowing for the separation of the axial and bending velocities. The trace from a single probe can then be compared with the pure axial velocity extracted from a double probe measurement. At an incident angle, $\theta$, of $10^{\circ}$, and careful alignment of the bar system, the $\sin \theta$ projection of bending velocities reduced the related errors below $1 \%$ in $12.7 \mathrm{~mm}$ bars (tested using input bar in the SHPB setup), and less for $25 \mathrm{~mm}$ bars. The reduction in the projection of bending modes allowed each bar to be probed with a single laser, reducing the number of velocimeter channels required from four to two.

Measurements are carried out at points along the bar surface, as depicted in Fig. 2, and are time shifted back to the bar faces. Probe positions are chosen to be sufficiently far for the stress wave to satisfy Saint-Venant's principle [8], making the stress uniform across the entire cross section of the bar, and thus making surface measurements representative of the bulk.

Applying the laser probe at glancing incidence causes the spot size of the laser on the bar to increase, and therefore the time resolution of the probe to decrease. Infra-red visualising card allowed the spot size at the surface to be measured to be of the order of a millimetre in diameter, which given a typical bar sound speed of over $1 \mathrm{~km} \mathrm{~s}^{-1}$ still allows for better than $1 \mu \mathrm{s}$ resolution.

\subsection{Striker bar measurements}

As the striker bar is repeatedly fired from the barrel, it is not coated in any kind of reflective or retro reflective paint. Instead the incident laser was shone at a steeper angle (typically $30^{\circ}$ ) to increase the diffuse return. Despite the absence of any paint, a signal was still collectable with the fibre placed $5 \mathrm{~mm}$ or closer to the bar surface. The laser probe was placed at the mid point of the bar, $12.5 \mathrm{~cm}$ from the sample firstly to satisfy Saint-Venant's principle as discussed above, and to aid wave separation, discussed in Sect. 3.1, as internal reflection in the striker requires additional wave separation after time shifting the trace to correspond to the face in contact with the sample.

\section{Method of analysis}

\subsection{Internal reflection in the striker bar}

Due to the nature and timescale of the unloading in the striker bar during direct impact tests, measurements of the sample face velocity will usually be superimposed with the tensile wave reflections of compressional waves returning from the free end of the bar. Separation of these waves becomes further complicated by bulk velocity, the velocity of the wave medium with reference to the probe. The velocity measured by the probe is the combination of three 
(b)

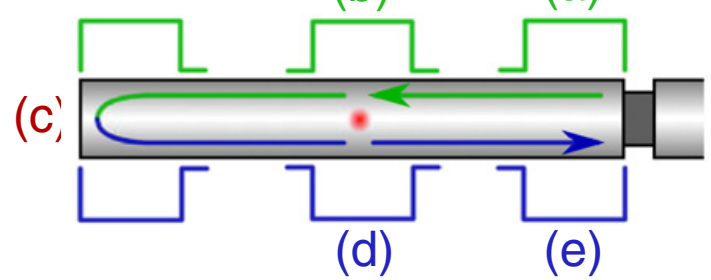

Figure 5. The complete path of a compression wave from the sample to the free face and back as a tensile wave, passing the measurement probe each way.

separate components,

$$
V_{\text {probe }}=V_{\text {bulk }}+V_{\text {compressive }}+V_{\text {tensile }} .
$$

In order to correctly restore the force and velocity history of the sample face, these profiles must be correctly separated and time shifted. Separation is possible as the initial bulk velocity is measurable, and the initial tensile velocity is zero.

Figure 5 depicts the complete path of one wave in the striker. The compression wave is formed at the sample, (a), at $t_{1}$ and passes by the measurement probe (b) at $t_{2}$. It then reaches the back surface (c) where the free end boundary condition forces it to be perfectly reflected into a tensile wave. The tensile wave passes back past the measurement probe at (d) at a time $t_{3}$ and contributes a further change in the velocity at that point, equal to that the compression wave created at (b). After passing as a reflected wave, the tensile wave returns to the sample at (e) and any effects of the wave at that point can then be considered as part of the motion at the sample face, and do not require separating and correcting.

The initial velocity at the struck face of the specimen is the signal in the absence of the reflected trace, as that is the only velocity not to originate from the sample. The corrected trace is then given by

$$
V_{s t}\left(t_{1}\right)=V_{\text {probe }}\left(t_{2}\right)-V_{\text {tensile }}\left(t_{2}\right),
$$

where $V_{s t}$ is the velocity of the struck face of the sample, and timeshifting from $t_{2}$ to $t_{1}$ is applied as discussed above. Given perfect reflections, the inversion of both wave type and travel direction at the striker free end means the velocity change due to the tensile component is the same as that of the equivalent earlier compressive component,

$$
V_{\text {tensile }}\left(t_{3}\right)=V_{\text {compressive }}\left(t_{2}\right) \text {. }
$$

For the purpose of discussing the correction procedure simply, we will define a dimensionless time such that one unit of dimensionless time is the time taken for a sound wave to travel one length of the striker bar,

$$
\tau_{i}=\frac{c_{s t r}}{l_{s t r}} t_{i}
$$

where $\mathrm{c}_{s t r}$ is the sound speed and $l_{s t r}$ is the length of the striker bar. For a probe at the midpoint of the striker bar, the dimensionless time between $\tau_{1}$ and $\tau_{2}$ is 0.5 and between $\tau_{2}$ and $\tau_{3}$ is 1 . (a)

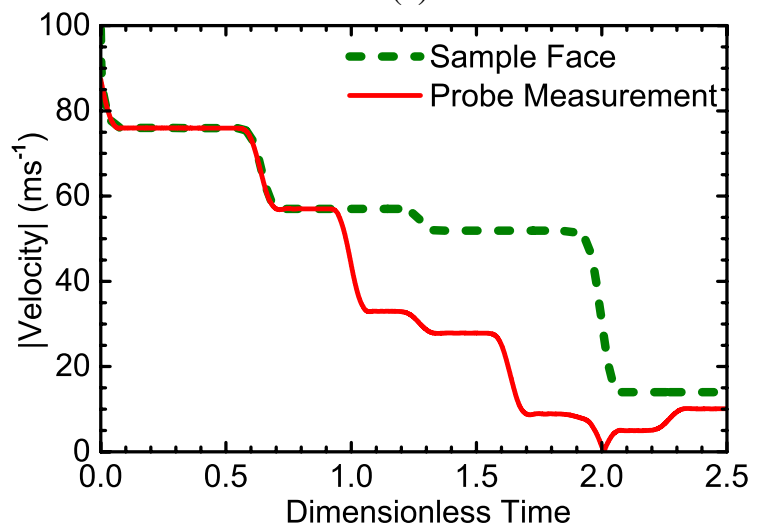

(b)

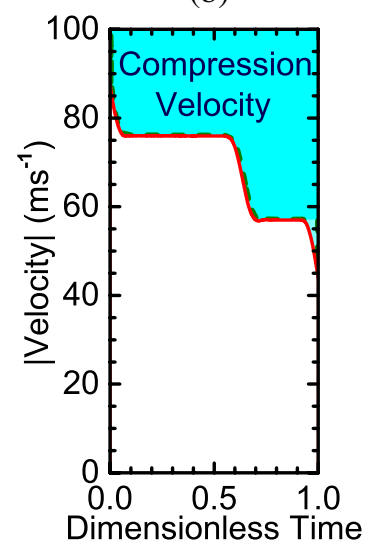

(c)

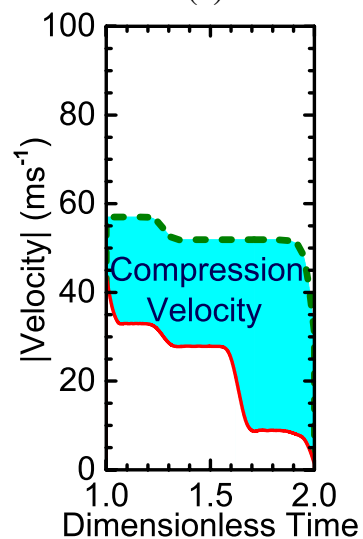

(d)

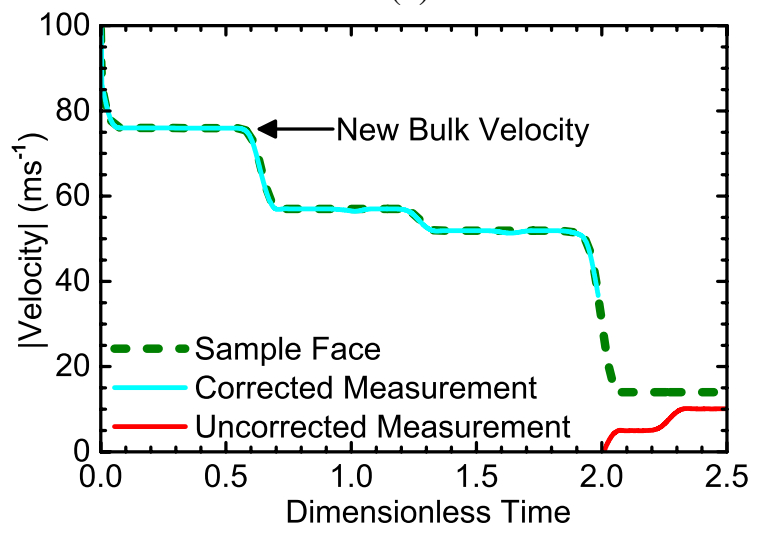

Figure 6. A series of "cartoon" velocity traces explaining how the correction procedure is performed. (a) depicts the uncorrected trace, (b) and (c) show the correction, and (d) shows the trace for the next unit of dimensionless time. The initial striker velocity is $100 \mathrm{~m} \mathrm{~s}^{-1}$.

Figure 6 depicts the correction procedure. In (a), both the probe measurement (solid line) and the true face velocity (dashed line) are shown. The probe trace has been time shifted back by $\tau=0.5$ to overlap it onto the corresponding sample face velocity. During the first unit of dimensionless time, the trace does not need any correction, as no tensile waves have returned. Using this correct measurement of the face velocity, and knowledge of the bulk velocity, the compressional velocity can be found for the first unit of dimensionless time, shown by the shaded 
area in Fig. 6 (b). The compressional velocity is in the opposite direction to the bulk velocity, making it negative if the bulk velocity is considered positive.

In Fig. 6 (c), the next segment of dimensionless time is corrected by subtracting the previously measured compressional velocity, assuming perfect reflection, to account for it returning as a tensile velocity. As the measured compressional velocity was negative, subtracting it from the later trace increases the velocity above the measured velocity. Practically this results in adding the shaded area from (b) on to the top of the probe measurement in (c). This gives the corrected trace as depicted in (d), from which the new bulk velocity of the bar can be determined.

The bulk velocity of a bar for any unit of dimensionless time is equal to last completed velocity step of the sample face in the previous unit of dimensionless time, as shown in Fig. 6 (d). This is due to completed steps in velocity being the ones which have traversed the entire bar, from (a) to (e) in Fig. 5.

Time shifting can be done more reliably in the case of striker reflections than reflections in the input bar in an SHPB, as the shifting takes place between parts of the same continuous wave; any incorrect shifting produces obvious spikes in the recovered velocity.

\subsection{Sample deformation calculation}

Once measurements have been made of the front, or striker impact face, $V_{s t}$, and the back, or output bar face, $V_{o p}$, the sample length as a function of time is determined by the difference between these two velocities

$$
\frac{\partial L(t)}{\partial t}=V_{o p}-V_{s t},
$$

and

$$
\varepsilon(t)=\ln \left(\frac{L_{0}}{L(t)}\right),
$$

where compressive strains are considered positive.

The stress history of the sample is measured as per a classic DIPB test, using the elastic relation

$$
\sigma(t)=V_{o p}(t) Z_{o p} \frac{A_{o p}}{A_{s a}(t)}
$$

where $\mathrm{Z}_{o p}$ is the acoustic impedance of the output bar and $\mathrm{A}_{o p}$ and $\mathrm{A}_{s a}$ are the cross sectional areas of the output bar and sample respectively.

\subsection{Force measurements and equilibrium}

The force at the output face can be found directly from the measured velocity

$$
F_{o p}(t)=V_{o p}(t) Z_{o p} A_{o p},
$$

the same relationship holds true for the striker bar. However, the only velocity component due to the force from the sample is the compressive wave

$$
F_{s t}(t)=Z_{\text {str }} A_{\text {str }} V_{\text {compressive }}(t) \text {. }
$$

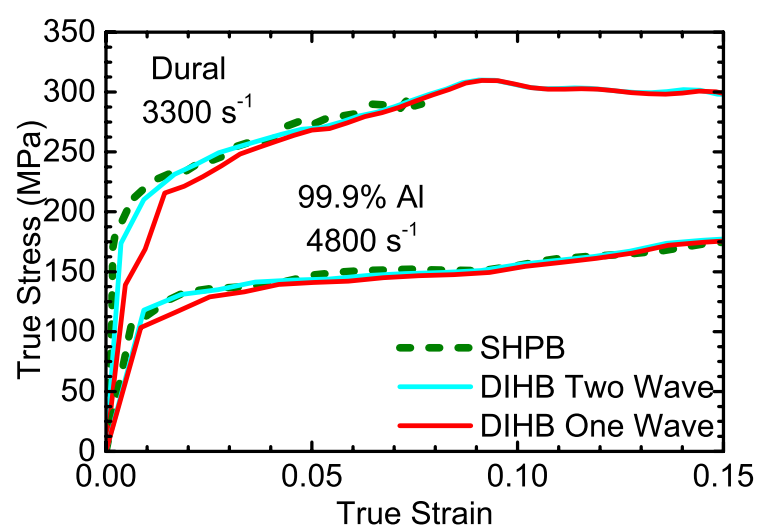

Figure 7. The stress-strain relationships for equal striker velocitiy tests in SHPB and DIHB setups. Two-wave analysis of the data appears to be a better match to SHPB data early on. However, one-wave measurements reach similar accuracy after a true strain of approximately 0.05 . The largest SHPB strain reached by the $99,9 \% \mathrm{Al}$ is 0.15 .

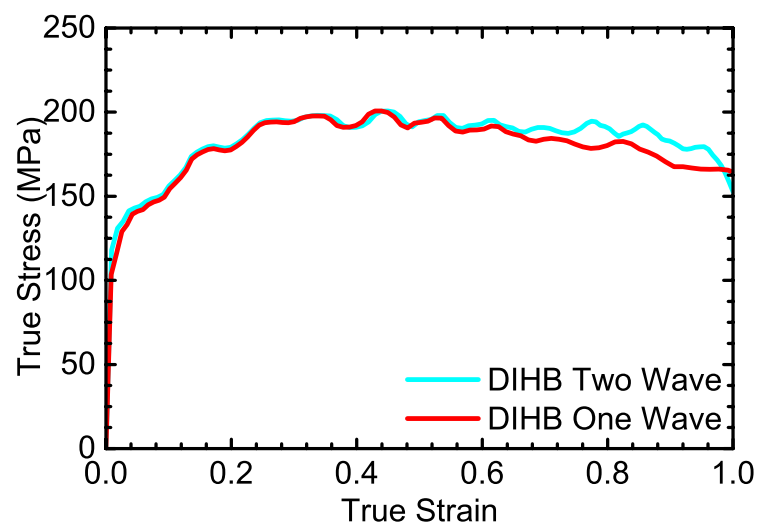

Figure 8. The stress strain relationships derived from one and two-wave measurements of the same DIHB test on $99.9 \% \mathrm{Al}$ at $4800 \mathrm{~s}^{-1}$. One-wave measurements show a notable drop in measured stress at high strain due to an overestimate of the striker face velocity and thus an overestimate of the sample area when conserving volume.

These measurements allow force equilibrium analysis to be performed in exactly the same way as classic split bar tests. It should be noted that bars of equal acoustic impedance and area, a compressional velocity comparison is equivalent to a force comparison.

\section{Experimental results \& discussion}

Sample disks of $1 \mathrm{~mm}$ thickness and $3 \mathrm{~mm}$ diameter made from a $99.9 \%$ pure aluminium and a commercial Dural alloy and were tested at equal striker velocities in both SHPB and DIHB arrangements. SHPB velocity traces from the surface were analysed using standard three-wave methods [5]. DIHB data was analysed using the two-wave analysis described in Sect. 3, and traditional one-wave analysis [2] where the striker face velocity is approximated by

$$
V_{s t}(t)=V_{0}-V_{o p}(t)
$$

where $\mathrm{V}_{0}$ is the firing velocity of the striker.

The stress-strain relationships obtained using these methods are depicted in Figs. 7 and 8. Figure 7 is cropped 


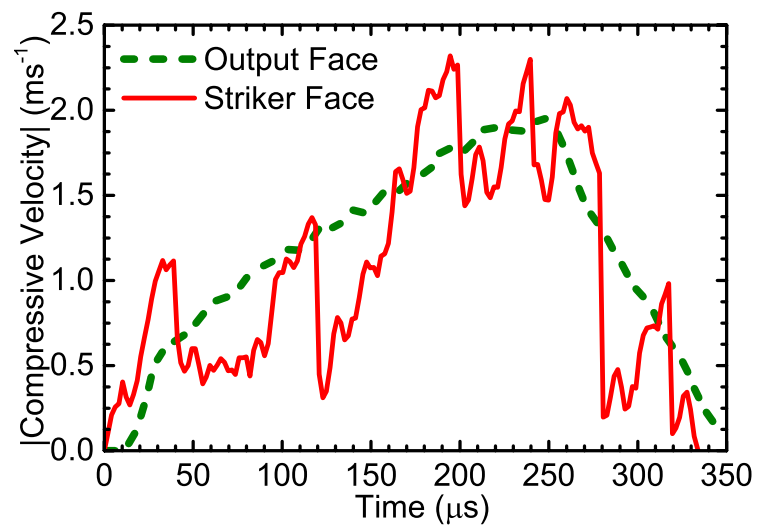

Figure 9. The compressional wave velocies leaving the sample through the striker bar and output bar as a function of time. These are directly proportional to the forces on the sample faces as shown in Eqs. (14) and (15). The spikes every $80 \mu \mathrm{s}$ in the striker face correspond to the wave in the striker bar making new passes of the sample after traveling two bar lengths (Striker Length $0.25 \mathrm{~m}$, sound speed $6.3 \mathrm{~km} \mathrm{~s}^{-1}$ ).

to a true strain of 0.2 to allow clearer comparison between the DIHB methods and the SHPB results. Complete curves for both DIHB methods are depicted in Fig. 8.

At true strains lower than 0.05, two-wave analysis of the DIHB data appears to be a closer match to SHPB data. This is likely due to the one-wave DIHB measurement incorrectly assuming force equilibrium during ring up in the sample. Figure 9 depicts the forces on the sample faces in the $99.9 \%$ aluminium DIHB test, approximately $10 \mu \mathrm{s}$ is taken for the back face of the sample to reach equilibrium with the front face. This time corresponds to a true strain of approximately 0.05 at a strain rate of $4800 \mathrm{~s}^{-1}$, making it similar to the time-scale over which the two-wave and one-wave traces differ.

In Fig. 8 for strains greater than 0.5 the one-wave trace is seen to consistently decrease below the twowave trace. By this point the bulk velocity of the striker bar has been reduced by multiple passes of decelerating waves. In one-wave analysis the striker's bulk velocity is assumed constant, as in Eq. (16). This leads to an over-estimation of strain and therefore sample area due to volume conservation, leading to an underestimate of the flow stress in Eq. (13).

\section{Conclusions}

We have presented a method for directly measuring the velocities and forces at the impacted face of a specimen in a DIHB experiment. Analysis of the data was demonstrated including the recovery of the sample face trace in the presence of free end striker reflections. Experimental data comparing the method to current approximations was presented with reference to equivalent SHPB data. Direct measurements were observed to more closely correspond to SHPB measurements for low strains and to not suffer from the same high strain inaccuracies as assuming constant striker inertia. Our method makes DIHB measurements viable for samples which do not quickly reach force equilibrium. These include polymers, foams and cellular materials, potentially allowing access to previously untested strain regimes.

This research was supported by grants from QinetiQ and the Engineering and Physical Sciences Research Council. The authors would like to thank P.J. Gould and P.D. Church for their interest.

\section{References}

[1] H. Kolsky, Proceedings of the Physical Society B 62, 676-700 (1949)

[2] D. Gorham, P. Pope, J. Field, Proceedings of the Royal Society A 438, 153-170 (1992)

[3] D. Gorham, Institute of Physics Conference Series 47, 16-24 (1980)

[4] R. Govender, Unpublished Presentation at DYMAT Hopkinson Centenary Conference (2014)

[5] C. Avinadav, Y. Ashuach, R. Kreif, Review of Scientific Instruments 82, 073908 (2011)

[6] O. Strand, D. Goosman, C. Martinez, T. Whitworth, W. Kuhlow, Review of Scientific Instruments 77, 083108 (2006)

[7] D. Casem, M. Zellner, Experimental Mechanics 53, 1467-1473 (2013)

[8] A. Love, A treatise on the mathematical theory of elasticity (Cambridge University Press, 1927) 257-258 